Schwarz Methods with Local Refinement for the p-Version Finite Element Method

Luca F. Pavarino

January, 1993

TR93-01 


\title{
Schwarz Methods with Local Refinement for the p-version Finite Element Method
}

\author{
Luca F. Pavarino * \\ January, 1993
}

\begin{abstract}
In some applications, the accuracy of the numerical solution of an elliptic problem needs to be increased only in certain parts of the domain. In this paper, local refinement is introduced for an overlapping additive Schwarz algorithm for the $p$-version finite element method. The resulting algorithm is highly parallel and scalable.

Under certain hypotheses on the refinement region, it is proved that in two and three dimensions the condition number of the iteration operator is bounded by a constant independent of $p$ and the number of subdomains. In the general two dimensional case, an almost optimal bound with logarithmic growth in $p$ is proved.
\end{abstract}

\section{Introduction.}

A domain decomposition method using $p$-version finite elements was analyzed in Pavarino [11], using the framework provided by the additive Schwarz method (ASM) of Dryja and Widlund [8], [9]. Due to the generous overlap between subdomains, we were able to show that the condition number of the ASM iteration operator is bounded by a constant independent of the degree $p$, the mesh size $H$ and the number of subdomains $N$. Here we consider a variant of the method based on local refinement. Our work is inspired by work of Bramble, Ewing, Parashkevov, and Pasciak, for the $h$-version finite element method, see [4], [5].

${ }^{*}$ Rice University, Department of Computational and Applied Mathematics, Houston, TX 77251. This work was supported by the National Science Foundation under grant NSF-CCR9120008, the U.S. Department of Energy under contract DE-FG-05-92ER25142 and the State of Texas under contract 1059. This work was also supported by the U.S. Department of Energy under contract DE-FG02-88ER25053 and by the National Science Foundation under Grant NSF-CCR-8903003, while the author was a student at the Courant Institute of Mathematical Science, 251 Mercer Street, New York, NY 10012. 
Local refinement algorithms can be of interest in many applications where the accuracy of the numerical solution needs to be increased only in certain parts of the domain. For standard $h$-version finite elements, local mesh refinement can be introduced by selecting and refining some elements of a coarse triangulation. This process can be applied recursively and multilevel methods can be considered. For the $p$-version finite element method considered here, local refinement amounts to increasing the degree $p$ of the polynomial basis functions only in selected elements of the fixed triangulation. We note that our results are easily extended to multiplicative variants of the algorithm by applying general theorems of the abstract Schwarz theory; see Dryja and Widlund [8], [9], Pavarino [12] and the references therein.

In Section 2 of this paper, we introduce a simple model problem and its discretization with the $p$-version finite element method. We then define an overlapping additive Schwarz algorithm, using local refinement, and formulate our main result in two dimensions. Under certain hypotheses on the boundary of the refinement region, we prove a constant bound for the condition number of the iteration operator, while in the general case, we prove an almost optimal logarithmic bound. The proof is based on some preliminary results, of independent interest, about discrete harmonic polynomials. The three dimensional case is studied in Section 3, while in Section 4 we illustrate our results with some numerical experiments.

\section{An additive Schwarz method with local refine- ment in two dimensions.}

For simplicity, we consider a linear, self adjoint, second order elliptic, model problem on a bounded Lipschitz region $\Omega \subset R^{2}$ :

Find $u \in V=H_{0}^{1}(\Omega)$ such that

$$
a(u, v)=f(v), \quad \forall v \in V,
$$

where the bilinear form

$$
a(u, v)=\int_{\Omega} \nabla u \cdot \nabla v d x
$$

defines a semi-norm $|u|_{H^{1}(\Omega)}=(a(u, u))^{1 / 2}$ in $H^{1}(\Omega)$, and a norm in $V=H_{0}^{1}(\Omega)$.

The discrete problem is given by the $p$-version finite element method. A triangulation of the region $\Omega$ is introduced by dividing it into non-overlapping quadrilateral elements $\Omega_{i}, i=1, \cdots, N_{e}$. We suppose, for simplicity, that the elements are squares of side $H$ and that the original region is a union of such elements. Using affine mappings onto a reference square, our analysis also works for general quadrilateral elements. Let $N$ be the number of interior mesh points. 
With refinement everywhere, we have the finite element space

$$
V^{p}=\left\{\phi \in C^{0}(\Omega):\left.\phi\right|_{\Omega_{i}} \in Q_{p}, i=1, \cdots, N_{e}\right\} \bigcap H_{0}^{1}(\Omega),
$$

where

$$
Q_{p}=\operatorname{span}\left\{x^{i} y^{j}: 0 \leq i, j \leq p\right\} .
$$

In the case of local refinement, we select $N^{r}<N$ interior nodes. Let $I^{r}$ be the set of refinement indexes. With each selected interior node $x_{i}$, we associate a subdomain $\Omega_{i}^{\prime}$, defined as the $2 H \times 2 H$ open square centered at $x_{i}$. The region of refinement is then

$$
\Omega_{r}=\bigcup \Omega_{i}^{\prime}, \quad i \in I^{r}
$$

and the finite element space

$$
V_{r}^{p}=V^{H}+V_{1}^{p}+\cdots+V_{N_{r}}^{p} .
$$

$V^{H}=V^{1}$ is the analog of the $h$-version coarse space and consists of the continuos functions defined on $\Omega$ that are piecewise bilinear on each element and vanish on $\partial \Omega . V_{i}^{p}=V^{p} \cap H_{0}^{1}\left(\Omega_{i}^{\prime}\right)$ are the local spaces.

The discrete problem is then:

Find $u_{p}^{*} \in V_{r}^{p}$ such that

$$
a\left(u_{p}^{*}, v_{p}\right)=f\left(v_{p}\right), \quad \forall v_{p} \in V_{r}^{p} .
$$

As before, the algorithm consists in solving, by an iterative method such as the conjugate gradient, the equation

$$
P u_{p}=\left(P_{0}+P_{1}+\ldots+P_{N_{r}}\right) u_{p}=g_{p},
$$

where the projections $P_{i}: V_{r}^{p} \rightarrow V_{i}^{p}$ are defined by

$$
a\left(P_{i} v_{p}, \phi_{p}\right)=a\left(v_{p}, \phi_{p}\right), \quad \forall \phi_{p} \in V_{i}^{p}
$$

For two dimensions, the main result of this paper is:

Theorem 1 Let $\Omega \subset R^{2}$. The operator $P$ of the additive algorithm defined by the spaces $V_{i}^{p}$ satisfies the estimate

$$
\kappa(P) \leq \text { const. }
$$

if there are no isolated points on $\partial \Omega_{r}$, and

$$
\kappa(P) \leq C(1+\log p)^{2}
$$

otherwise. 
A point on $\partial \Omega_{r}$ is isolated if it is not a limit (accumulation) point of $\partial \Omega_{r}$; see Section 4 for numerical experiments and examples. Before proving this theorem, we need to develop some technical results concerning the decomposition of discrete harmonic polynomials as well as review some classical tools in approximation theory. A polynomial $w \in Q_{p}$ is discrete harmonic on an element $\Omega_{i}$ if

$$
a(w, \phi)=0, \quad \forall \phi \in Q_{p} \text { that vanish on } \partial \Omega_{i} .
$$

A piecewise polynomial function $w \in V^{p}$ defined on $\Omega$ is discrete harmonic if it is so on each element.

\subsection{Auxiliary results}

When working with Sobolev norms on domains of diameter $O(H)$, we need to work with scaled norms. These are obtained by a change of variable from the standard definitions on a domain of diameter $O(1)$. For example,

$$
\begin{gathered}
\|u\|_{H^{1}(\Omega)}^{2}=|u|_{H^{1}(\Omega)}^{2}+\frac{1}{H^{2}}\|u\|_{L^{2}(\Omega)}^{2}, \\
\|u\|_{H^{1 / 2}(\partial \Omega)}^{2}=|u|_{H^{1 / 2}(\partial \Omega)}^{2}+\frac{1}{H}\|u\|_{L^{2}(\partial \Omega)}^{2} .
\end{gathered}
$$

We will use the classical Markov's Theorem:

Theorem 2 If $v$ is a polynomial of degree $p$ on $I=[-1,1]$, then

$$
\max _{I}\left|v^{\prime}(x)\right| \leq p^{2} \max _{I}|v(x)|
$$

with equality only for $v= \pm T_{p}$, the Chebyshev polynomial of degree $p$. This inequality is not scale invariant: on the interval $I_{H}=[-H, H]$

$$
\max _{I_{H}}\left|v^{\prime}(x)\right| \leq \frac{p^{2}}{H} \max _{I_{H}}|v(x)|
$$

A proof and many useful generalizations can be found in Rivlin [14]. The following result is Lemma 2.2 in Bramble and $\mathrm{Xu}$ [6]:

Lemma 1 If $D$ is a bounded Lipschitz domain in $R^{2}$, then

$$
\|w\|_{L^{\infty}(D)} \leq C\left(|\log \epsilon|^{1 / 2}\|w\|_{H^{1}(D)}+\epsilon\|w\|_{W^{1, \infty}(D)}\right)
$$

for every function $w \in W^{1, \infty}(D)$ and any $\epsilon \in(0,1)$.

With this Lemma, we can prove a Sobolev-like inequality for polynomial finite element: 
Lemma 2 If $u_{p} \in Q_{p}\left(\Omega_{i}\right)$, where $\Omega_{i}$ is an element of diameter $H$, then

$$
\left\|u_{p}\right\|_{L^{\infty}\left(\Omega_{i}\right)}^{2} \leq C(1+\log p)\left\|u_{p}\right\|_{H^{1}\left(\Omega_{i}\right)}^{2} .
$$

Proof. Apply Lemma 1 to $w=u_{p}$ on one element $D=\Omega_{i}$. Then

$$
\left\|u_{p}\right\|_{L^{\infty}} \leq C\left(|\log \epsilon|^{1 / 2}\left\|u_{p}\right\|_{H^{1}}+\epsilon\left\|u_{p}\right\|_{W^{1, \infty}}\right) .
$$

On an element $\Omega_{i}$ of diameter $H$, the $W^{1, \infty}$-norm is given by

$$
\left\|u_{p}\right\|_{W^{1, \infty}\left(\Omega_{i}\right)}=\left\|u_{p}\right\|_{L^{\infty}\left(\Omega_{i}\right)}+H\left\|\nabla u_{p}\right\|_{L^{\infty}\left(\Omega_{i}\right)} .
$$

By Markov's inequality:

$$
\left\|u_{p}\right\|_{W^{1, \infty}\left(\Omega_{i}\right)} \leq\left(1+2 H \frac{p^{2}}{H}\right)\left\|u_{p}\right\|_{L^{\infty}\left(\Omega_{i}\right)}=\left(1+2 p^{2}\right)\left\|u_{p}\right\|_{L^{\infty}\left(\Omega_{i}\right)} .
$$

Choosing $\epsilon=\frac{1}{6 C p^{2}}$ in Lemma 1, we obtain:

$$
\left\|u_{p}\right\|_{L^{\infty}\left(\Omega_{i}\right)} \leq C|\log 6 C+2 \log p|^{1 / 2}\left\|u_{p}\right\|_{H^{1}\left(\Omega_{i}\right)}+\frac{1+2 p^{2}}{6 p^{2}}\left\|u_{p}\right\|_{L^{\infty}\left(\Omega_{i}\right)}
$$

and finally

$$
\left\|u_{p}\right\|_{L^{\infty}\left(\Omega_{i}\right)}^{2} \leq C(1+\log p)\left\|u_{p}\right\|_{H^{1}\left(\Omega_{i}\right)}^{2}
$$

Corollary 1 If $u_{p}$ of Lemma 2 vanishes at some point in $\Omega_{i}$, then

$$
\left\|u_{p}\right\|_{L^{\infty}\left(\Omega_{i}\right)}^{2} \leq C(1+\log p)\left\|u_{p}+\alpha\right\|_{H^{1}\left(\Omega_{i}\right)}^{2},
$$

for every constant $\alpha$.

Proof. Use the inequality

$$
\left\|u_{p}\right\|_{\infty} \leq\left\|u_{p}+\alpha\right\|_{\infty}+\|\alpha\|_{\infty} \leq 2\left\|u_{p}+\alpha\right\|_{\infty}
$$

and apply Lemma 2 .

Corollary 2 If $\beta$ is any value of $u_{p}$ in $\Omega_{i}$, then

$$
\left\|u_{p}-\beta\right\|_{L^{\infty}\left(\Omega_{i}\right)}^{2} \leq C(1+\log p)\left|u_{p}\right|_{H^{1}\left(\Omega_{i}\right)}^{2} .
$$

Proof. Consider the inf over $\alpha$ in the estimate of Corollary 1. By a quotient space argument, we obtain

$$
\left\|u_{p}\right\|_{L^{\infty}}^{2} \leq C(1+\log p)\left|u_{p}\right|_{H^{1}}^{2} .
$$

Then, just apply this estimate to $u_{p}-\beta$. 
One of our main tools is the following $p$-version analog of Lemma 3.2 in Widlund [15]:

Lemma 3 Let $w \in Q_{p}$ be a discrete harmonic polynomial on a square element $\Omega_{i}$, that vanishes at the vertices of $\Omega_{i}$. Then there exist four discrete harmonic polynomials $w_{j} \in Q_{p}$, each with nonzero boundary values only on one side $\Gamma_{j}$ of $\Omega_{i}$, such that $w=\sum_{j} w_{j}$ and

$$
\sum_{j=1}^{4}\left|w_{j}\right|_{H^{1 / 2}\left(\partial \Omega_{i}\right)}^{2} \leq C(1+\log p)^{2}|w|_{H^{1}\left(\Omega_{i}\right)}^{2}
$$

Proof. For each side $\Gamma_{j}$, we define

$$
w_{j}=\left\{\begin{array}{l}
w \text { on } \Gamma_{j} \\
0 \text { on } \Gamma_{i} \neq \Gamma_{j} \\
\text { discrete harmonic extension in } \Omega_{i} .
\end{array}\right.
$$

Clearly $w=\sum_{j} w_{j}$. By definition

$$
\left|w_{j}\right|_{H^{1 / 2}\left(\partial \Omega_{i}\right)}^{2}=\int_{\partial \Omega_{i}} \int_{\partial \Omega_{i}} \frac{\left|w_{j}(x(s))-w_{j}\left(x\left(s^{\prime}\right)\right)\right|^{2}}{\left|x(s)-x\left(s^{\prime}\right)\right|^{2}} d s^{\prime} d s .
$$

Since $\partial \Omega_{i}=\bigcup_{k} \bar{\Gamma}_{k}$, we have:

$$
\left|w_{j}\right|_{H^{1 / 2}\left(\partial \Omega_{i}\right)}^{2}=\left|w_{j}\right|_{H^{1 / 2}\left(\Gamma_{j}\right)}^{2}+\sum_{k \neq j} \int_{\Gamma_{j}} \int_{\Gamma_{k}} \frac{|w(x(s))|^{2}}{\left|x(s)-x\left(s^{\prime}\right)\right|^{2}} d s^{\prime} d s .
$$

The first term can be bounded by using the trace Theorem:

$$
\left|w_{j}\right|_{H^{1 / 2}\left(\Gamma_{j}\right)}^{2}=|w|_{H^{1 / 2}\left(\Gamma_{j}\right)}^{2} \leq|w|_{H^{1 / 2}\left(\partial \Omega_{i}\right)}^{2} \leq C|w|_{H^{1}\left(\Omega_{i}\right)}^{2} .
$$

Now consider one of the other terms. If $\Gamma_{k}$ and $\Gamma_{j}$ are opposite sides of $\Omega_{i}$, then $\left|x(s)-x\left(s^{\prime}\right)\right| \geq H$. We then parametrize and bound the double integral by

$$
\int_{0}^{H} \int_{0}^{H} \frac{|w(x(s))|^{2}}{\left|x(s)-x\left(s^{\prime}\right)\right|^{2}} d s^{\prime} d s \leq \frac{1}{H} \int_{0}^{H}|w(x(s))|^{2} d s \leq\|w\|_{L^{\infty}\left(\Omega_{i}\right)}^{2} .
$$

By Corollary 2 , this is bounded by $C(1+\log p)|w|_{H^{1}\left(\Omega_{i}\right)}^{2}$. If $\Gamma_{k}$ and $\Gamma_{j}$ share a vertex of $\Omega_{i}$, we parametrize the double integral as

$$
\int_{0}^{H} \int_{-H}^{0} \frac{|w(x(s))|^{2}}{\left|x(s)-x\left(s^{\prime}\right)\right|^{2}} d s^{\prime} d s
$$


This integral can be estimated by

$$
2 \int_{0}^{H} \int_{-H}^{0} \frac{|w(x(s))|^{2}}{\left|s-s^{\prime}\right|^{2}} d s^{\prime} d s \leq 2 \int_{0}^{H} \frac{|w(x(s))|^{2}}{s} d s
$$

because

$$
\int_{-H}^{0} \frac{d s^{\prime}}{\left(s-s^{\prime}\right)^{2}}=\left.\frac{1}{s-s^{\prime}}\right|_{-H} ^{0}=\frac{1}{s}-\frac{1}{s+H} \leq \frac{1}{s} .
$$

We divide each resulting integral into two:

$$
\int_{0}^{H} \frac{|w(x(s))|^{2}}{s} d s=\int_{0}^{H / p^{2}} \frac{|w(x(s))|^{2}}{s} d s+\int_{H / p^{2}}^{H} \frac{|w(x(s))|^{2}}{s} d s .
$$

We estimate the first term by using the mean value Theorem and Markov's inequality.

$$
\begin{gathered}
\int_{0}^{H / p^{2}} \frac{|w(x(s))|^{2}}{s} d s=\int_{0}^{H / p^{2}}\left|\frac{d w}{d s}(\tilde{x})\right|^{2} \frac{(x(s)-x(0))^{2}}{s} d s \leq \\
\leq\left(\frac{p^{2}}{H}\right)^{2}\|w\|_{L^{\infty}\left(\Omega_{i}\right)}^{2} \int_{0}^{H / p^{2}} \frac{(x(s)-x(0))^{2}}{s} d s=\left(\frac{p^{2}}{H}\right)^{2}\|w\|_{L^{\infty}\left(\Omega_{i}\right)}^{2} \int_{0}^{H / p^{2}} s d s= \\
=\left(\frac{p^{2}}{H}\right)^{2}\|w\|_{L^{\infty}\left(\Omega_{i}\right)}^{2} \frac{1}{2}\left(\frac{H}{p^{2}}\right)^{2}=\frac{1}{2}\|w\|_{L^{\infty}\left(\Omega_{i}\right)}^{2},
\end{gathered}
$$

since $x(s)-x(0)=s$. We estimate the second term by using the discrete Sobolev inequality of Corollary 2.

$$
\begin{aligned}
\int_{H / p^{2}}^{H} \frac{|w(x(s))|^{2}}{s} d s & \leq\|w\|_{L^{\infty}\left(\Omega_{i}\right)}^{2} \log \frac{H}{H / p^{2}}=2 \log p\|w\|_{L^{\infty}\left(\Omega_{i}\right)}^{2} \leq \\
& \leq C(1+\log p)^{2}|w|_{H^{1}\left(\Omega_{i}\right)}^{2}
\end{aligned}
$$

Combining these estimates, we obtain the result.

We will need the following two dimensional extension Theorem for polynomial finite element functions. The proof, for a square and a triangle, can be found in Babuška et al. [1] or Bernardi and Maday [3]. Using results of Canuto and Funaro [7], it is possible to prove a three dimensional polynomial extension Theorem, see Belgacem [2] and Pavarino and Widlund [13].

Theorem 3 Let $\Omega_{i}$ be a square element and $f \in H^{1 / 2}\left(\partial \Omega_{i}\right)$ be a polynomial of degree $p$ on each side of $\Omega_{i}$, which is continuous on $\partial \Omega_{i}$. Then there exists a polynomial $u \in Q_{p}\left(\Omega_{i}\right)$ such that $u=f$ on $\partial \Omega_{i}$ and

$$
\|u\|_{H^{1}\left(\Omega_{i}\right)} \leq C\|f\|_{H^{1 / 2}\left(\partial \Omega_{i}\right)} .
$$




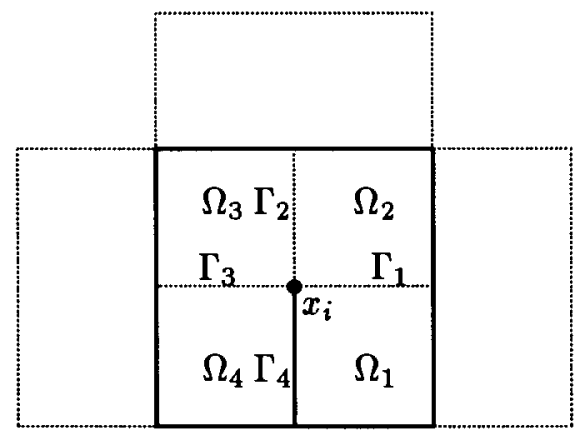

Figure 1: Decomposition of $w_{i}$ in 2-dim

Using this extension Theorem, it is possible to prove the following $p$-version analog of Lemma 4.1 of Bramble et al. [5]. Lemma 4 is a generalization of Lemma 3 of Pavarino [11].

Lemma 4 Consider an interior node $x_{i}$, with its associated subdomain $\Omega_{i}^{\prime}$, and let $\Gamma_{j}, j=1,2,3,4$, denote the four edges connecting $x_{i}$ and $\partial \Omega_{i}^{\prime}$ (see Figure 1). Let $w_{i} \in V_{i}^{p}=V^{p} \bigcap H_{0}^{1}\left(\Omega_{i}^{\prime}\right)$ be a discrete harmonic polynomial that vanishes at the interior node $x_{i}$. Let $w_{i j}$ be the discrete harmonic polynomial that equals $w_{i}$ on $\Gamma_{j}$ and vanishes on the remaining edges.

Then $w_{i}=\sum_{j=1}^{4} w_{i j}$ and

$$
\sum_{j=1}^{4} a\left(w_{i j}, w_{i j}\right) \leq C a\left(w_{i}, w_{i}\right)
$$

if $w_{i}$ vanishes on at least one of the $\Gamma_{j}$, and

$$
\sum_{j} a\left(w_{i j}, w_{i j}\right) \leq C(1+\log p)^{2} a\left(w_{i}, w_{i}\right)
$$

otherwise.

Proof. a) Let suppose that $w_{i}=0$ on the edge $\Gamma_{4}$. We start by constructing $w_{i 1}$ with support in $\Omega_{1} \cup \Omega_{2} \cup \Gamma_{1}$ :

$$
w_{i 1}=\left\{\begin{array}{l}
w_{i} \text { on } \Omega_{1} \cup \Gamma_{1} \\
\text { reflection across } \Gamma_{1} \text { from } \Omega_{1} \text { onto } \Omega_{2} .
\end{array}\right.
$$

Clearly $w_{i 1}$ is discrete harmonic, since $w_{i}$ is, and

$$
\left|w_{i 1}\right|_{H^{1}\left(\Omega_{i}^{\prime}\right)}=2\left|w_{i 1}\right|_{H^{1}\left(\Omega_{1}\right)}=2\left|w_{i}\right|_{H^{1}\left(\Omega_{1}\right)} \leq 2\left|w_{i}\right|_{H^{1}\left(\Omega_{i}^{\prime}\right)}
$$


Now $w_{i}-w_{i 1}$ is discrete harmonic, vanishes on $\Gamma_{1}$ and, by the triangle inequality, $\left|w_{i}-w_{i 1}\right|_{H^{1}\left(\Omega_{i}^{\prime}\right)} \leq 3\left|w_{i}\right|_{H^{1}\left(\Omega_{i}^{\prime}\right)}$. We can therefore construct $w_{i 2}$ with support in $\Omega_{2} \cup \Omega_{3} \cup \Gamma_{2}$ in the same way:

$$
w_{i 2}=\left\{\begin{array}{l}
w_{i}-w_{i 1} \text { on } \Omega_{2} \cup \Gamma_{2} \\
\text { reflection across } \Gamma_{2} \text { from } \Omega_{2} \text { onto } \Omega_{3}
\end{array}\right.
$$

$w_{i 2}$ is discrete harmonic, $\left|w_{i 2}\right|_{H^{1}\left(\Omega_{i}^{\prime}\right)} \leq 6\left|w_{i}\right|_{H^{1}\left(\Omega_{i}^{\prime}\right)}$ and $\left|w_{i}-w_{i 1}-w_{i 2}\right|_{H^{1}\left(\Omega_{i}^{\prime}\right)} \leq$ $9\left|w_{i}\right|_{H^{1}\left(\Omega_{i}^{\prime}\right)}$. Similarly, we construct $w_{i 3}$ with support in $\Omega_{3} \cup \Omega_{4} \cup \Gamma_{3}$ :

$$
w_{i 3}=\left\{\begin{array}{l}
w_{i}-w_{i 1}-w_{i 2} \text { on } \Omega_{3} \cup \Gamma_{3} \\
\text { reflection across } \Gamma_{3} \text { from } \Omega_{3} \text { onto } \Omega_{4} .
\end{array}\right.
$$

Again, $w_{i 3}$ is discrete harmonic and $\left|w_{i 3}\right|_{H^{1}\left(\Omega_{j}^{\prime}\right)} \leq 18\left|w_{i}\right|_{H^{1}\left(\Omega_{i}^{\prime}\right)}$.

Finally, we define $w_{i 4}=w_{i}-\sum_{j} w_{i j}$ and it is clear that $w_{i 4}$ is discrete harmonic, with support in $\Omega_{4}$ and that $\left|w_{i 4}\right|_{H^{1}\left(\Omega_{i}^{\prime}\right)} \leq 27\left|w_{i}\right|_{H^{1}\left(\Omega_{i}^{\prime}\right)}$.

Therefore $\sum_{j=1}^{4} w_{i j}=w_{i}$ and $\sum_{j=1}^{4} a\left(w_{i j}, w_{i j}\right) \leq C a\left(w_{i}, w_{i}\right)$.

b) We suppose now that $w_{i}$ does not vanish identically on any $\Gamma_{j}$. Consider one of the four elements of $\Omega_{i}^{\prime}$, for example $\Omega_{1}$. $w_{i}$ vanishes on the two edges of $\Omega_{1}$ that are on the boundary of $\Omega_{i}^{\prime}$ and differs from zero on the other two interior edges $\Gamma_{1}$ and $\Gamma_{4}$. We want to write $w_{i}$ as a sum of two discrete harmonic functions each different from zero on only one of the interior edges. By Lemma 3 , we can find two discrete harmonic polynomials $w_{i 1}$ and $w_{i 4}$ with nonzero values only on $\Gamma_{1}$ and $\Gamma_{4}$, respectively, such that

$$
\left|w_{i 1}\right|_{H^{1 / 2}\left(\partial \Omega_{1}\right)}^{2}+\left|w_{i 4}\right|_{H^{1 / 2}\left(\partial \Omega_{1}\right)}^{2} \leq C(1+\log p)^{2}\left|w_{i}\right|_{H^{1}\left(\Omega_{1}\right)}^{2} .
$$

By the polynomial extension Theorem 3

$$
\left|w_{i 1}\right|_{H^{1}\left(\Omega_{1}\right)}^{2} \leq C\left|w_{i 1}\right|_{H^{1 / 2}\left(\partial \Omega_{1}\right)}^{2}
$$

Therefore

$$
\left|w_{i 1}\right|_{H^{1}\left(\Omega_{1}\right)}^{2} \leq C(1+\log p)^{2}\left|w_{i}\right|_{H^{1}\left(\Omega_{1}\right)}^{2}
$$

and the same inequality holds for $w_{i 4}$.

Repeating the process on the other elements in $\Omega_{i}^{\prime}$, we obtain eight discrete harmonic polynomials. Combining the pairs corresponding to the same interior edge, where they have the same values by definition, we finally obtain the four discrete harmonic piecewise polynomials with the desired properties. 


\subsection{Proof of Theorem 1}

We can now proceed to prove Theorem 1 . As in the case with refinement everywhere studied in [11], we base the proof on the Schwarz framework of Dryja and Widlund [8], [9].

A constant upper bound for the spectrum of $\mathrm{P}$ is obtained by noting that for $i \geq 1$

$$
a\left(P_{i} u_{p}, u_{p}\right)=a\left(P_{i} u_{p}, P_{i} u_{p}\right)=a_{\Omega_{i}^{\prime}}\left(P_{i} u_{p}, P_{i} u_{p}\right) \leq a_{\Omega_{i}^{\prime}}\left(u_{p}, u_{p}\right) .
$$

Each point is covered by no more than four subregions $\Omega_{i}^{\prime}$ and the norm of $P_{0}$ is equal to one. Therefore $\lambda_{\max } \leq 5$.

A lower bound is obtained by Lemma 1 in Pavarino [11], Lemma 4 in the previous Section and by defining a partition of $u \in V_{r}^{p}$ with the required properties. We start by defining $u_{0} \in V^{H}$, by specifying its values at the interior nodes $x_{i}$ :

$$
u_{0}\left(x_{i}\right)=\left\{\begin{array}{lr}
u\left(x_{i}\right) & \text { if } x_{i} \notin \Omega_{\tau} \\
Q_{H} u\left(x_{i}\right) & \text { if } x_{i} \in \Omega_{\tau}
\end{array}\right.
$$

Here $Q_{H}$ is the $L_{2}$-projection of $V_{T}^{p}$ onto $V^{H}$. It has the properties

$$
\begin{gathered}
\left\|\left(I-Q_{H}\right) v\right\|_{L^{2}(\Omega)}^{2} \leq C H^{2} a(v, v) \\
a\left(Q_{H} v, Q_{H} v\right) \leq C a(v, v)
\end{gathered}
$$

for every function $v \in H_{0}^{1}(\Omega)$, see Bramble and Xu [6]. Since $u-u_{0} \equiv 0$ outside $\Omega_{r}$, we have

$$
a_{\Omega}\left(u-u_{0}, u-u_{0}\right)=a_{\Omega_{r}}\left(u-u_{0}, u-u_{0}\right) .
$$

Using the triangle inequality, we have

$$
\begin{gathered}
a\left(u-u_{0}, u-u_{0}\right)^{1 / 2}=a_{\Omega_{r}}\left(u-Q_{H} u+Q_{H} u-u_{0}, u-Q_{H} u+Q_{H} u-u_{0}\right)^{1 / 2} \leq \\
a_{\Omega_{r}}\left(\left(I-Q_{H}\right) u,\left(I-Q_{H}\right) u\right)^{1 / 2}+a_{\Omega_{r}}\left(Q_{H} u-u_{0}, Q_{H} u-u_{0}\right)^{1 / 2} \\
\leq C a(u, u)^{1 / 2}+a_{\Omega_{r}}\left(Q_{H} u-u_{0}, Q_{H} u-u_{0}\right)^{1 / 2} .
\end{gathered}
$$

By definition $Q_{H} u-u_{0} \equiv 0$ in $\Omega_{\tau}$ except in those elements $\Omega_{i}$ which have at least one vertex on $\partial \Omega_{r}$. Therefore, expanding $Q_{H} u-u_{0}=\sum_{i}\left(Q_{H} u-u_{0}\right)\left(x_{i}\right) \phi_{i}$ in the nodal basis and using the properties of $Q_{H}$, we obtain:

$$
\begin{gathered}
a_{\Omega_{r}}\left(Q_{H} u-u_{0}, Q_{H} u-u_{0}\right) \leq C \sum_{x_{i} \in \partial \Omega_{r}}\left(Q_{H} u-u_{0}\right)^{2}\left(x_{i}\right) \leq \\
\leq C H^{-2}\left\|Q_{H} u-u_{0}\right\|_{L^{2}(\Omega)}^{2} \leq C a(u, u) .
\end{gathered}
$$

We have then proved

$$
a\left(u-u_{0}, u-u_{0}\right) \leq C a(u, u)
$$


Reasoning in the same way, we prove

$$
\left\|u-u_{0}\right\|_{L^{2}(\Omega)}^{2} \leq C H^{2} a(u, u)
$$

We now need to decompose $w=u-u_{0} \in H_{0}^{1}\left(\Omega_{r}\right)$. We use a result from Pavarino [11] (see eqn. (24)) for the case of refinement everywhere:

There exists a decomposition $w=\sum_{i=1}^{N} w_{i}$ such that

$$
\sum_{i=1}^{N} a\left(w_{i}, w_{i}\right) \leq C\left(a(w, w)+\frac{1}{H^{2}}\|w\|_{L^{2}(\Omega)}^{2}\right)
$$

and $\operatorname{supp}\left(w_{i}\right) \subset \operatorname{supp}(w)$.

Here $N$ is the number of all interior nodes in $\Omega$. Therefore this is not the desired decomposition, since it contains terms $w_{i}$ with $i \notin I_{r}$, which correspond to $x_{i} \notin \Omega_{r}$. Since $\operatorname{supp}\left(w_{i}\right) \subset \operatorname{supp}(w)$, these remaining terms $w_{i}$ vanish unless $x_{i} \in \partial \Omega_{r}$. Therefore, we need to decompose only the terms $w_{i}$ corresponding to $x_{i} \in \partial \Omega_{r}$ and distribute their contributions among the other terms. First, we write

$$
w_{i}=w^{0}+y
$$

where $y=w_{i}$ on the mesh lines and is extended as a discrete harmonic polynomial inside each element. $w^{0}=w_{i}-y$ vanishes on the mesh lines and is $a$-orthogonal to $y$. Thus

$$
a\left(w_{i}, w_{i}\right)=a\left(w^{0}, w^{0}\right)+a(y, y)
$$

Since $w^{0}$ is nonzero only on elements in $\Omega_{r}$, we can regard these elements as belonging to a unique subdomain $\Omega_{j}^{\prime}$ with $j \in I_{r}$ and add the restrictions of $w^{0}$ on these elements to the corresponding term $w_{j}$. We still denote these modified terms by $w_{j}$. We now use Lemma 4 to decompose $y$. If there are no isolated points on $\partial \Omega_{r}$, there is at least one edge of the mesh belonging to $\partial \Omega_{r}$ ending at $x_{i}$. Since $w_{i}=0$ on this edge, $y$ will vanish there too. Therefore, by Lemma $4, y=\sum_{j} y_{j}$ and

$$
\sum_{j} a\left(y_{j}, y_{j}\right) \leq C a(y, y)
$$

If $x_{i}$ is an isolated point of $\partial \Omega_{r}$, then Lemma 4 will give us a decomposition of $y$ satisfying the weaker inequality:

$$
\sum_{j} a\left(y_{j}, y_{j}\right) \leq C(1+\log p)^{2} a(y, y) .
$$

The functions $y_{j}$ are nonzero only on one edge of the mesh and this edge is assigned uniquely to a subdomain $\Omega_{k}^{\prime}$ in $\Omega_{r}$. We add the functions $y_{j}$ to the 
corresponding $w_{k}$ and still denote the modified terms by $w_{k}$. We then have found a decomposition

$$
w=\sum_{i \in I_{r}} w_{i}
$$

satisfying

$$
\sum_{i \in I_{r}} a\left(w_{i}, w_{i}\right) \leq C\left(a(w, w)+\frac{1}{H^{2}}\|w\|_{L^{2}(\Omega)}^{2}\right)
$$

in the case without isolated points on $\partial \Omega_{r}$ and

$$
\sum_{i \in I_{r}} a\left(w_{i}, w_{i}\right) \leq C(1+\log p)^{2}\left(a(w, w)+\frac{1}{H^{2}}\|w\|_{L^{2}(\Omega)}^{2}\right)
$$

in the general case. Using (4) and (5), we can conclude that the decomposition of $u$ satisfies the inequality of Lemma 1 in Pavarino [11] with a constant independent of $p$ and $N_{r}$, in the case without isolated points, and with $C(1+\log p)^{2}$ otherwise. A lower bound of the smallest eigenvalue of the iteration operator $P$ is then observed.

\section{An overlapping ASM with local refinement in three dimensions}

We now consider regular refinement regions, in the sense specified in Theorem 4 , and establish an optimal bound for $\kappa(P)$. The general case still requires further research.

Our model problem is given on a region $\Omega \subset R^{3}$, which for simplicity is the union of non-overlapping cubic elements $\Omega_{i}$ with mesh size $H$. Homogeneous Dirichlet boundary conditions are given on $\partial \Omega$. With the same notation as in the two dimensional case, we associate with each selected interior node $x_{i}$ a subdomain $\Omega_{i}^{\prime}$ defined as the open cube of side $2 H$ centered at $x_{i}$. The refinement region is

$$
\Omega_{r}=\bigcup \Omega_{i}^{\prime}, \quad i \in I^{r}
$$

and the finite element space is decomposed as

$$
V_{r}^{p}=V^{H}+V_{1}^{p}+\cdots+V_{N_{r}}^{p}
$$

where $V^{H}=V^{1}$ and $V_{i}^{p}=V^{p} \cap H_{0}^{1}\left(\Omega_{i}^{\prime}\right)$ are the local spaces. The definition of the algorithm in terms of projections is the same as before. The main result is: 
Theorem 4 Let $\Omega \subset R^{3}$. If there are no isolated points or edges on the boundary $\partial \Omega_{\tau}$ of the refinement region, then the operator $P$ of the ASM defined by the spaces $V_{i}^{p}$ satisfies the estimates

$$
\kappa(P) \leq \text { const. }
$$

Proof. We proceed as in the two dimensional proof of the constant upper bound and in the construction of the coarse component $u_{0}$ in the decomposition of $u \in V_{r}^{p}$. We then apply to $w=u-u_{0}$ the three dimensional decomposition result proved in Pavarino [11] for the case of refinement everywhere:

There exists a decomposition $w=\sum_{i=1}^{N} w_{i}$ such that

$$
\sum_{i=1}^{N} a\left(w_{i}, w_{i}\right) \leq C\left(a(w, w)+\frac{1}{H^{2}}\|w\|_{L^{2}(\Omega)}^{2}\right)
$$

and $\operatorname{supp}\left(w_{i}\right) \subset \operatorname{supp}(w)$.

This is not the desired decomposition, since it contains terms with $i \notin I_{\tau}$. As before, we need to decompose only the terms $w_{i}$ corresponding to $x_{i} \in \partial \Omega_{r}$. We write

$$
w_{i}=w^{0}+y
$$

where $y=w_{i}$ on the interface $\Gamma=\bigcup \partial \Omega_{i}$ and is extended as a discrete harmonic polynomial inside each element. $w^{0}=w_{i}-y$ vanishes on the interface and is $a$-orthogonal to $y$ :

$$
a\left(w_{i}, w_{i}\right)=a\left(w^{0}, w^{0}\right)+a(y, y) .
$$

Since $w^{0}$ is nonzero only on elements in $\Omega_{r}$, it can be distributed as in the two dimensional case. We now decompose and distribute $y$. If there are no isolated points or edges on $\partial \Omega_{\tau}$, then there is at least one face of the interface belonging to $\partial \Omega_{r}$ which contains $x_{i}$. Since $w_{i}=0$ on this face, $y$ will vanish there too. We then apply the following Lemma and conclude as in the proof of Theorem 1:

Lemma 5 Let $x_{i}$ be an interior node with its associated subdomain $\Omega_{i}^{\prime}$ (union of eight elements $\Omega_{i}$; see Figure 2). Let $F_{i j}=\bar{\Omega}_{i} \cap \overline{\Omega_{j}}$ be the face between $\Omega_{i}$ and $\Omega_{j}$ and let $x_{i k}, k=1, \cdots, 6$, be the central nodes of the faces of $\Omega_{i}^{\prime}$. Let $w_{i} \in V_{i}^{p}=V^{p} \cap H_{0}^{1}\left(\Omega_{i}^{\prime}\right)$ be a discrete harmonic polynomial that vanishes at the interior node $x_{i}$ and assume that $w_{i}$ also vanishes on at least one face $F_{i j}$. Then there exist discrete harmonic polynomials $w_{i k} \in V_{i k}^{p}, k=1, \cdots, 6$, such that $w_{i}=\sum_{k} w_{i k}$ and

$$
\sum_{k=1}^{6} a\left(w_{i k}, w_{i k}\right) \leq C a\left(w_{i}, w_{i}\right)
$$




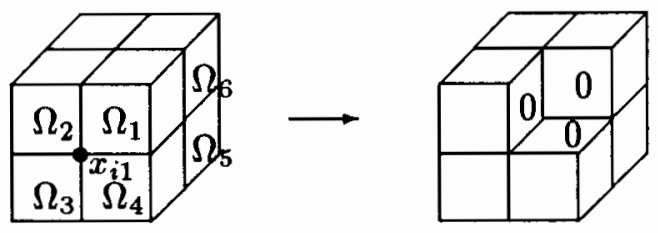

Figure 2: First step in the decomposition of $w_{i}$ in 3-dim

Proof. Let us suppose that $w_{i}=0$ on the face between $\Omega_{1}$ and $\Omega_{6}$ (see Figure 2 ) and denote by $x_{i 1}$ the interior node shared by the elements $\Omega_{1}, \Omega_{2}, \Omega_{3}$, and $\Omega_{4}$. We construct $w_{i 1} \in V_{i 1}^{p}$ by reflection across faces:

$$
w_{i 1}=\left\{\begin{array}{llll}
w_{i 1} \text { in } \Omega_{1} \cup F_{12} \cup F_{14} & \\
\text { reflection across } & F_{12} & \text { from } \bar{\Omega}_{1} \text { onto } \bar{\Omega}_{2} \\
\text { reflection across } & F_{14} & \text { from } \bar{\Omega}_{1} \text { onto } \bar{\Omega}_{4} \\
\text { reflection across } & F_{23} & \text { from } \bar{\Omega}_{2} \text { onto } \bar{\Omega}_{3}
\end{array}\right.
$$

Clearly $w_{i 1} \in V_{i 1}^{p}$ and

$$
\left|w_{i 1}\right|_{H^{1}\left(\Omega_{i 1}^{\prime}\right)}=4\left|w_{i 1}\right|_{H^{1}\left(\Omega_{1}\right)}=4\left|w_{i}\right|_{H^{1}\left(\Omega_{1}\right)} \leq 4\left|w_{i}\right|_{H^{1}\left(\Omega_{i}^{\prime}\right)} .
$$

$w_{i}-w_{i 1}$ vanishes on $F_{12}$ and $F_{14}$ and it has its support in $\Omega_{i}^{\prime} \backslash \Omega_{1}$. This subregion is formed by seven cubic elements, see Figure 2 . We now observe that this is the case treated in Lemma 3.4 of Pavarino [12]. Using this Lemma, we can further decompose $w_{i}-w_{i 1}$ into the remaining terms $w_{i k}$ with the desired properties.

\section{Numerical experiments in two dimensions}

In this section, we describe some numerical experiments in two dimensions with the local refinement $p$-method. For similar experiments with the $h$-version, see Bramble, Ewing, Parashkevov, and Pasciak [5]. More complete experiments for the $h$-version, with parallel machines and different methods can be found in Moe [10].

The programs, written in MATLAB, have been run on Sun Sparc workstations. In each set of experiments, we consider the Poisson equation in two dimensions with homogeneous Dirichlet boundary conditions. The equation 
$P u_{p}=g_{p}$ is solved using the conjugate gradient method. The iteration process is stopped when the relative $l^{2}$-norm of the residual has been decreased by at least $10^{-9}$. In the following tables, $\kappa(P)=\lambda_{\max } / \lambda_{\min }$ is the condition number of the operator $P$, computed by the Lanczos method.

1) We consider first a problem with no isolated points on $\partial \Omega_{r}$. This is a case of regular refinement.

$$
\left\{\begin{aligned}
-\Delta u & =-\frac{\pi^{2}}{8} \sin \left(\frac{\pi}{4} x\right) \sin \left(\frac{\pi}{4} y\right) . & & \text { in } \Omega \\
u & =0 & & \text { on } \partial \Omega
\end{aligned}\right.
$$

The square region $\Omega=[0,8]^{2}$ has 16 elements and 5 refinement subregions, see Figure 3. The condition number and the number of iterations are reported in Table 1. In this case, $\lambda_{\max }$ is constant and $\lambda_{\min }$ seems to converge as $p$ increases.

2) We next consider a case of irregular refinement for problem 1 . This selection of refinement points leaves an isolated point on $\partial \Omega_{r}$, see Figure 4 . From the results of Table 2, we see that $\lambda_{\max }$ is still constant, but that $\lambda_{\min }$ decreases considerably. A least square approximation shows that the condition number grows like $C(\log p)^{2}$.

3) Finally, we consider a problem with an isolated edge on the boundary of $\Omega_{r}$, see Figure 5. Since in this case we do not have isolated points on $\partial \Omega_{r}$, this is a regular case and the theory predicts that the condition number is bounded by a constant. This is confirmed by the results of Table 3 . 


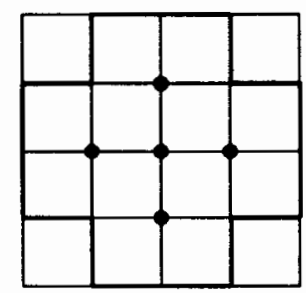

Figure 3: Regular choice of refinement points

\begin{tabular}{|c|c|c|c|c|}
\hline degree $\boldsymbol{p}$ & $\kappa(P)$ & $\lambda_{\max }$ & $\lambda_{\min }$ & iter. \\
\hline $\mathbf{3}$ & 5.7256 & $\mathbf{3}$ & $\mathbf{0 . 5 2 4 0}$ & 12 \\
4 & 5.9885 & $\mathbf{3}$ & 0.5010 & 14 \\
5 & 6.1371 & $\mathbf{3}$ & 0.4888 & 15 \\
6 & 6.2328 & $\mathbf{3}$ & $\mathbf{0 . 4 8 1 3}$ & 16 \\
7 & 6.2986 & $\mathbf{3}$ & $\mathbf{0 . 4 7 6 3}$ & 16 \\
$\mathbf{8}$ & 6.3466 & $\mathbf{3}$ & 0.4727 & 17 \\
\hline
\end{tabular}

Table 1: Regular refinement for pb. 1

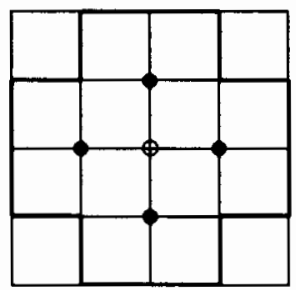

Figure 4: Irregular choice of refinement points 


\begin{tabular}{|c|c|c|c|c|}
\hline degree $p$ & $\kappa(P)$ & $\lambda_{\max }$ & $\lambda_{\min }$ & iter. \\
\hline 3 & 8.1493 & 2 & 0.2454 & 12 \\
4 & 10.4374 & 2 & 0.1916 & 13 \\
5 & 12.4814 & 2 & 0.1602 & 13 \\
6 & 14.3318 & 2 & 0.1395 & 14 \\
7 & 16.0255 & 2 & 0.1248 & 14 \\
8 & 17.5900 & 2 & 0.1137 & 15 \\
\hline
\end{tabular}

Table 2: Irregular refinement for pb. 2

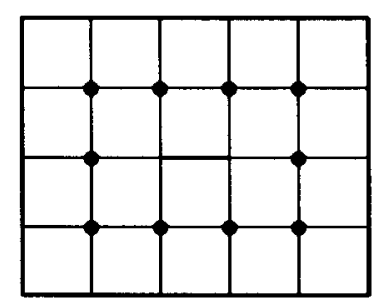

Figure 5: Refinement points with an isolated edge on $\partial \Omega_{r}$

\begin{tabular}{|c|c|c|c|c|}
\hline degree $p$ & $\kappa(P)$ & $\lambda_{\max }$ & $\lambda_{\min }$ & iter. \\
\hline 3 & 9.0330 & 3 & 0.3321 & 22 \\
4 & 10.0987 & 3 & 0.2971 & 23 \\
5 & 10.8288 & 3 & 0.2770 & 23 \\
6 & 11.2881 & 3 & 0.2658 & 24 \\
7 & 11.6386 & 3 & 0.2578 & 24 \\
8 & 11.8852 & 3 & 0.2524 & 25 \\
\hline
\end{tabular}

Table 3: Regular refinement for pb. 3

Acknowledgments. I would like to thank Prof. Olof Widlund for suggesting this problem and for all the help and time he has devoted to my work. 


\section{References}

[1] Ivo Babuška, Alan Craig, Jan Mandel, and Juhani Pitkäranta. Efficient preconditioning for the p-version finite element method in two dimensions. SIAM J. Numer. Anal., 28(3):624-661, 1991.

[2] F. B. Belgacem. Opérateurs de relèvements polynomiaux sur un cube. Technical Report HI-72/7780, E.D.F./D.E.F., 1992.

[3] Christine Bernardi and Yvon Maday. Relèvement polynomial de traces et applications. $M^{2} A N, 24(5): 557-611,1990$.

[4] J. Bramble, R. Ewing, R. Parashkevov, and J. Pasciak. Domain decomposition methods for problems with uniform local refinement in two dimensions. In R. Glowinski, Y. Kuznetsov, G. Meurant, J. Périaux, and O. Widlund, editors, Fourth International Symposium on Domain Decomposition Methods for Partial Differential Equations, Philadelphia, 1991. SIAM.

[5] J. Bramble, R. Ewing, R. Parashkevov, and J. Pasciak. Domain decomposition methods for problems with partial refinement. SIAM J. Sci. Stat. Comput., 13:397-410, 1992.

[6] James H. Bramble and Jinchao Xu. Some estimates for a weighted $L^{2}$ projection. Math. Comp., 56:463-476, 1991.

[7] C. Canuto and D. Funaro. The Schwarz algorithm for spectral methods. SIAM J. Numer. Anal., 25(1):24-40, 1988.

[8] Maksymilian Dryja and Olof B. Widlund. Additive Schwarz methods for elliptic finite element problems in three dimensions. In Tony F. Chan, David E. Keyes, Gérard A. Meurant, Jeffrey S. Scroggs, and Robert G. Voigt, editors, Fifth Conference on Domain Decomposition Methods for Partial Differential Equations, Philadelphia, PA, 1992. SIAM.

[9] Maksymilian Dryja and Olof B. Widlund. Schwarz methods of NeumannNeumann type for three-dimensional elliptic finite element problems. Technical report, Department of Computer Science, Courant Institute, 1993. In preparation.

[10] Randi Moe. Iterative Local Uniform Mesh Refinement Methods and Parallel Processing. PhD thesis, Department of Informatics, University of Bergen, Norway, June 1992.

[11] Luca F. Pavarino. An additive Schwarz method for the p-version finite element method. Technical Report 580, Courant Institute of Mathematical 
Sciences, Department of Computer Science, September 1991. A revised version has been submitted to Numer. Math.

[12] Luca F. Pavarino. Domain Decomposition Algorithms for the p-version finite element method for elliptic problems. PhD thesis, Courant Institute, New York University, September 1992.

[13] Luca F. Pavarino and Olof B. Widlund. Iterative substructuring methods for p-version finite elements in three dimensions. Technical report, Courant Institute of Mathematical Sciences, Department of Computer Science, 1993. To appear.

[14] T. J. Rivlin. Chebyshev Polynomials. Wiley Interscience, 1990.

[15] Olof B. Widlund. Iterative substructuring methods: Algorithms and theory for elliptic problems in the plane. In Roland Glowinski, Gene H. Golub, Gérard A. Meurant, and Jacques Périaux, editors, First International Symposium on Domain Decomposition Methods for Partial Differential Equations, Philadelphia, PA, 1988. SIAM. 